\title{
Automatic Detection and Labelling of the Human Cortical Folds in Magnetic Resonance Data Sets
}

\author{
Gabriele Lohmann, D. Yves von Cramon \\ Max-Planck-Institute of Cognitive Neuroscience \\ Inselstr. 22 - 26, 04103 Leipzig, Germany \\ Ph: ++49-341-9940 217, Fax: ++49-341-9940 221 \\ email: lohmann,cramon@cns.mpg.de
}

\begin{abstract}
The folding of the cortical surface of the human brain varies dramatically from person to person. However, the folding pattern is not arbitrary. The cortical folds (also called "sulci") often serve as landmarks for referencing brain locations, and the most pronounced sulci have names that are well established in the neuroanatomical literature. In this paper, we will present a method that both automatically detects and attributes neuroanatomical names to these folds using image analysis methods applied to magnetic resonance data of human brains. More precisely, we subdivide each fold into a number of substructures which we call sulcal basins, and attach labels to these basins. These sulcal basins form a complete parcellation of the cortical surface.

The algorithm reported here is important in the context of human brain mapping. Human brain mapping aims at establishing correspondences between brain function and brain anatomy. One of the most intriguing problems in this field is the high inter-personal variability of human neuroanatomy which makes studies across many subjects very difficult. Most previous attempts at solving this problem are based on various methods of image registration where MR data sets of different subjects are warped until they overlap. We believe that in the process of warping too much of the individual anatomy is destroyed so that relevant information is lost. The approach presented in this paper allows inter-personal comparisons without having to resort to image warping. Our concept of sulcal basins allows to establish a complete parcellation of the cortical surface into separate regions. These regions are neuroanatomically meaningful and can be identified from MR data sets across many subjects. At the same time, the parcellation is detailed enough to be useful for brain mapping purposes.
\end{abstract}

\section{Introduction}

The folding of the cortical surface of the human brain varies dramatically from person to person. However, the folding pattern is not arbitrary. In fact, the cortical folds (also called "sulci") often serve as landmarks for referencing brain locations, and the more pronounced sulci have names that are well established in the neuroanatomical literature [1]. 
In this paper, we will present a method that both automatically detects and attributes neuroanatomical names to these folds. More precisely, we subdivide each fold into a number of substructures which we call sulcal basins, and attach labels to these substructures. The reason why we introduce the concept of a sulcal basin is that we believe that sulcal basins have a lower degree of interpersonal variability than entire sulci.

Our method is important in the context of human brain mapping. Human brain mapping aims at establishing correspondences between brain function and brain anatomy. One of the most intriguing problems in this field is the high inter-personal variability of human neuroanatomy which makes studies across many subjects very difficult.

Most previous attempts at solving this problem are based on various methods of image registration where MR data sets are registered and warped onto a brain atlas [2],[3],[4],[5],[6]. A related approach is that by Gueziec et al. [7] and also Declerck et al. [8] who presented methods for extracting and matching lines in multiple MR data sets. However, in the process of warping much of the individual anatomy is destroyed by geometric distortions so that relevant information is lost. In particular, size and shape information is destroyed.

The approach presented in this paper allows inter-personal comparisons without having to resort to image warping. Our concept of sulcal basins allows to establish a complete parcellation of the cortical surface into separate regions. These regions are neuroanatomically meaningful and can be identified from MR data sets across many subjects. At the same time, the parcellation is detailed enough to be useful for brain mapping purposes.

The work closest in spirit to ours is that by Mangin et al. [9],[10],[11] who also seek to obtain a structural description of the cortical topography. It differs from ours in that they do not use use the concept of a sulcal basin which is fundamental to our approach. Instead, they use an approach based on structural decompositions of sulcal skeletons.

The paper is organized as follows. We begin by defining the concept of a sulcal basin and present an algorithm for extracting sulcal basins from MR images of the human brain. We then introduce a brain model consisting of sulcal basins and their spatial relationships. Finally, we present a graph matching approach that performs an automatic labelling of the sulcal basins.

\section{Sulcal basins}

\subsection{The concept of a sulcal basin}

The notion of a sulcal basin has not been used in the literature before. Let us therefore begin by defining this concept. Figure la shows a volume rendering of a MR data set depicting a top right view of a healthy subject's brain. The sulci are clearly visible as dark valleys. Figure $1 \mathrm{~b}$ shows the top part of the same brain. This time however, we removed the grey matter so that the white matter surface becomes visible and the sulci become more pronounced. Corresponding locations in both images are indicated by labels. 


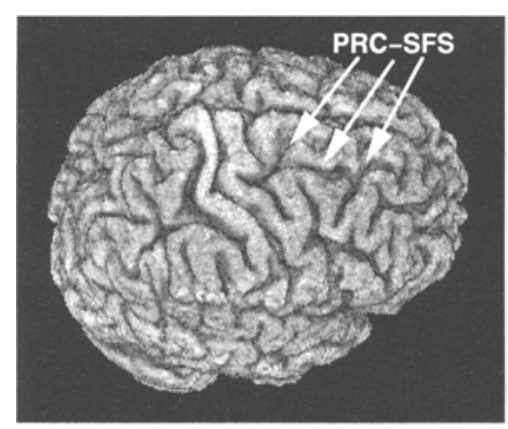

a) volume rendering

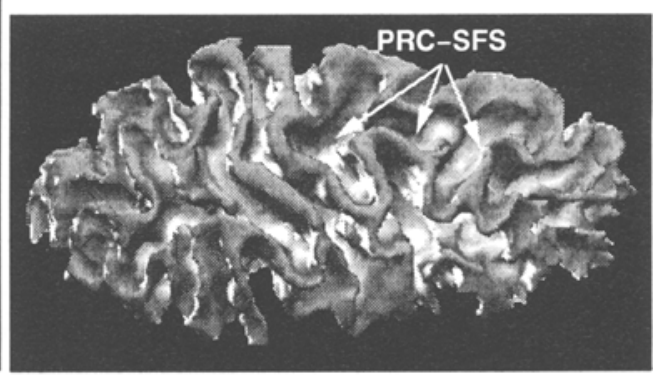

b) sulcal basins

Figure 1: Sulcal basins

Note that the fold labelled "prc-sfs (precentral/superior frontal sulcus)" which appears to consist of one large part in the volume rendering decomposes into three separate concave basins in the figure $1 \mathrm{~b}$. In fact, all sulci decompose into several such substructures, which we call "sulcal basins".

More precisely, sulcal basins are defined to be concavities in the white matter surface which are bounded by convex ridges that separate one basin from the next so that adjacent sulcal basins meet at the top of the ridge. Figure 2 illustrates this definition. The entire white matter surface is covered by such concavities so that a decomposition into sulcal basins yields a complete parcellation of the surface.

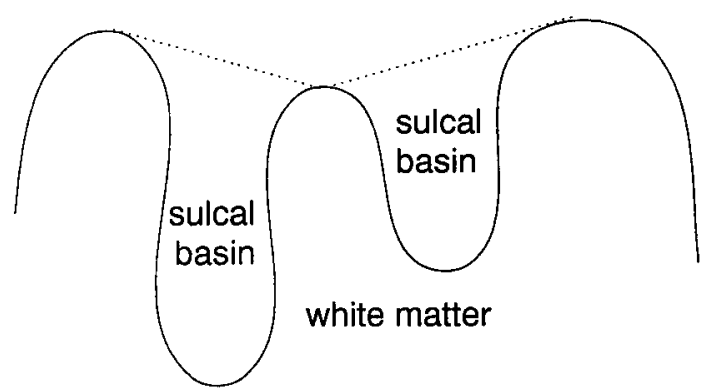

Figure 2: Sulcal basins

There are two principal advantages in introducing the concept of a sulcal basin. Firstly, in subdividing sulci into atomic parts we obtain a spatially more precise definition of brain loci. As we are ultimately interested in inter-subject comparisons, this is an important consideration.

Secondly, the high interpersonal variability in sulcal patterns can at least be partly attributed to different forms of groupings of sulcal basins. The two sets of sulcal basins below for instance can be easily matched, even though the two groups formed in each set cannot be matched:
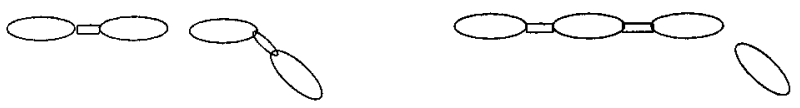
The post-central sulcus for instance usually consists of two basins, a superior and an inferior basin. In some brains, these two basins are connected to form a coherent sulcus. In others, there are completely disconnected. Thus, sulcal basins are much more useful as entities for matching than entire sulci.

\subsection{Automatic detection of sulcal basins from magnetic resonance data sets}

Several approaches to automatic sulcus detection from MR images have been reported in the literature [12], [13], [14] which either seek surface representations of the cortical folds or are based on skeletonization methods.

The concept of sulcal basins is new, and consequently no methods for extracting them from MR images have existed so far. In the following, we will describe our approach.

Sulcal basins are concave indentations in the white matter surface, so that in principle it would be possible to detect sulcal basins by simply computing curvature properties of the white matter surface. Parts of the surface which belong to a sulcal basin are concave so that both the mean curvature and the Gaussian curvature are positive. Boundaries between basins are either saddles or ridges and have negative or zero curvature values.

However, the white matter surface is highly convoluted and the MR data sets have a limited spatial resolution, so that the computation of second order differentials becomes quite inaccurate. As a consequence, we found that curvature computations are not feasible for our purpose. In addition, there are also some parts of a sulcal basin wall which are convex and yet they do not constitute a boundary between basins as illustrated below:

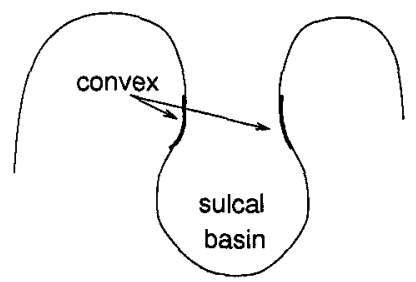

Therefore, we use a different approach which is not based on curvature properties. The method consists of a sequence of image analysis steps of which the first four are illustrated in figure 3 .

The input data set (fig $3 \mathrm{a}$ ) is first subjected to a white matter segmentation which separates white matter from other tissue classes (fig. 3b). This step helps to make the sulcal indentations more pronounced and thus more easily identifiable.

A large number of segmentation algorithms are known from the literature. Any suitable segmentation procedure can be used here. A general segmentation algorithm that produces satisfactory results for all types of input data does not exist at this point, so that the choice of a suitable algorithm and its parameters still very much depends on the type of data at hand. In our experiments, we 
used both simple thresholding techniques, as well as a new algorithm based on region growing [15].

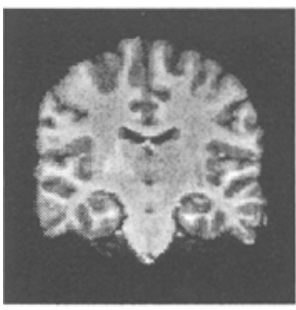

a) MR image

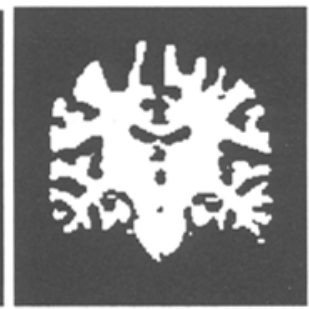

b) white matter

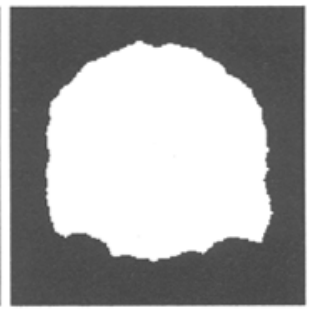

c) closing

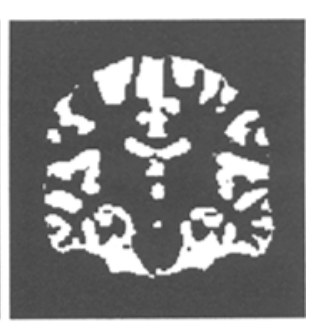

d) sulcal interiors

Figure 3: The first four steps of the algorithm

We then close the sulci using a 3D morphological closing filter [16] to obtain an idealized smoothed surface (fig. 3c). We use a structuring element of spherical shape with a very large diameter. The exact size of the diameter is not critical as long as it is large enough. We subtract the white matter from the morphologically closed image so that only the sulcal interiors remain (fig. $3 \mathrm{~d}$ ).

At this point in the procedure, the processed image contains the union of all sulcal basins. We now need to separate the individual basins by trying to find the ridges between them. The main idea is based on the observation that each sulcal basin has a locally deepest point (or a small set of locally deepest points) which may be used to guide the search.

The sulcal depth is computed with respect to the smoothed surface (fig. 3c) using a 3D distance transform [17]. The distance transform attaches a value to each white voxel which encodes its distance towards the nearest black voxel so that each sulcal interior point of figure $3 \mathrm{~d}$ receives a depth label. In essence, we first try to detect small "islands" of locally deepest points assuming that each such island represents one sulcal basin.

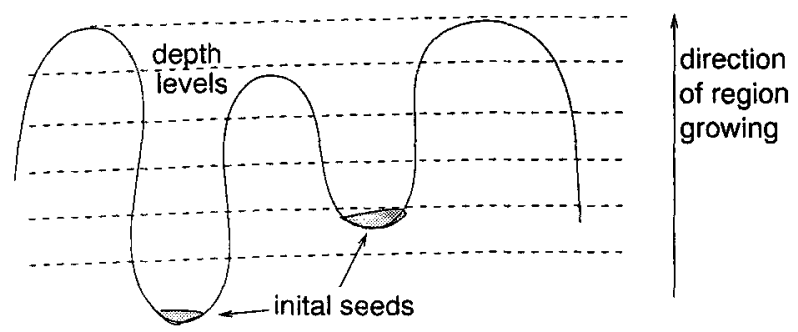

Figure 4: Region growing

We search for locally deepest points by moving a search window of size $n \times$ $n \times n$ across the image where typically $n=7$. Adjoining deepest points that are of almost equal depth and are close are merged into one larger patch so that perturbations due to noise are eliminated.

Finally, we let each initial island grow in a manner similar to region growing until a separating ridge is encountered. During region growing, successively higher levels are processed so that at each stage in the procedure only voxels of 
a given depth are added to a sulcal basin. Figure 4 illustrates this process, and figure 5 shows a data flow diagram of the entire procedure.

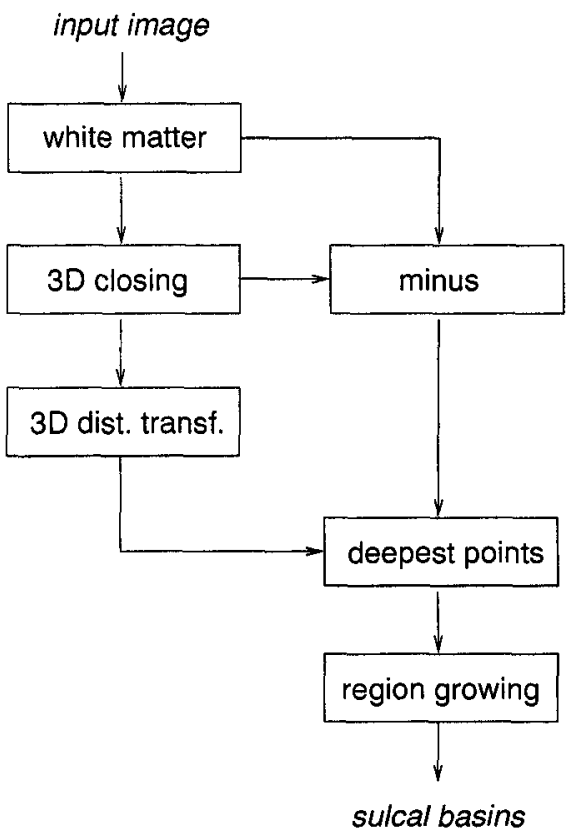

Figure 5: Flow diagram

\section{Knowledge representation}

In the following, we will describe a method of automatically attaching neuroanatomical labels to the sulcal basins that have been identified using the procedures described in the previous sections. We will begin by describing our knowledge representation scheme.

The neuroanatomical knowledge we use for describing sulcal basins consists of two parts: firstly, we use a unary model that describes unary predicates of a sulcal basin such as location, depth or size. Secondly, we use a relational model that describes spatial relationships between basins.

The unary model was obtained as follows. We first rotate all data sets into a standard coordinate system where the origin is taken to be half-way between CA (commissura anterior) and CP (commissura posterior) making our coordinate system analogous to the one proposed in [18].

We then performed a hand labelling of four data sets of which the sulcal basins had been extracted beforehand. We identified a set of 29 neuroanatomical labels which are listed in table $\mathbf{I}$.

These labels were chosen because they represent all primary and secondary sulci, covering the most part of the lateral brain surface. The structures that are not represented here are the medial sulci which reside along the inter-hemispheric 
cleft, the basal sulci which are located along the bottom of the brain, the Sylvian fissure, and some tertiary sulci which have a high degree of interpersonal variability so that no well established neuroanatomical names exist for them.

Each sulcal basin of the above list is represented by its centroid, which is computed from the four hand-labelled data sets. Figure 6 shows these centroids against the sulcal pattern of one of these four data sets (the sulcal lines shown here for better orientation were extracted using the algorithm described in [19]).

\begin{tabular}{|r|l|l|}
\hline id & neuroanatomical name & abbreviation \\
\hline 1 & superior central sulcus & cs sup \\
2 & central sulcus broca's knee & cs broca \\
3 & inferior central sulcus & cs inf \\
4 & superior precentral sulcus & prc sup (fef) \\
5 & medial precentral sulcus & prc med \\
6 & inferior precentral sulcus & prc inf \\
7 & superior frontal sulcus 1 (posterior part) & sfs 1 \\
8 & superior frontal sulcus 2 & sfs 2 \\
9 & superior frontal sulcus 3 & sfs 3 \\
10 & superior frontal sulcus 4 (anterior part) & sfs 4 \\
11 & intermediate frontal sulcus (posterior part) & imfs 1 \\
12 & intermediate frontal sulcus (anterior part) & imfs 2 \\
13 & inferior frontal sulcus (posterior part) & ifs 1 (post) \\
14 & inferior frontal sulcus (anterior part) & ifs 2 (ant) \\
15 & superior post-central sulcus & poc sup \\
16 & inferior post-central sulcus & poc inf \\
17 & intraparietal sulcus (anterior) & ips asc \\
18 & intraparietal sulcus (medial) & ips hor \\
19 & intraparietal sulcus (descending) & ips desc \\
20 & superior temporal sulcus 1 (posterior part) & sts 1 (post) \\
21 & superior temporal sulcus 2 & sts 2 \\
22 & superior temporal sulcus 3 & sts 3 \\
23 & superior temporal sulcus 4 (anterior part) & sts 4 \\
24 & medial temporal sulcus & mts \\
25 & inferior temporal sulcus 1 (posterior part) & its 1 (post) \\
26 & inferior temporal sulcus 2 & its 2 \\
27 & inferior temporal sulcus 3 & its 3 \\
28 & inferior temporal sulcus 4 (anterior part) & its 4 \\
29 & occipital sulcus & occ \\
\hline
\end{tabular}

Table I: list of neuroanatomical labels 


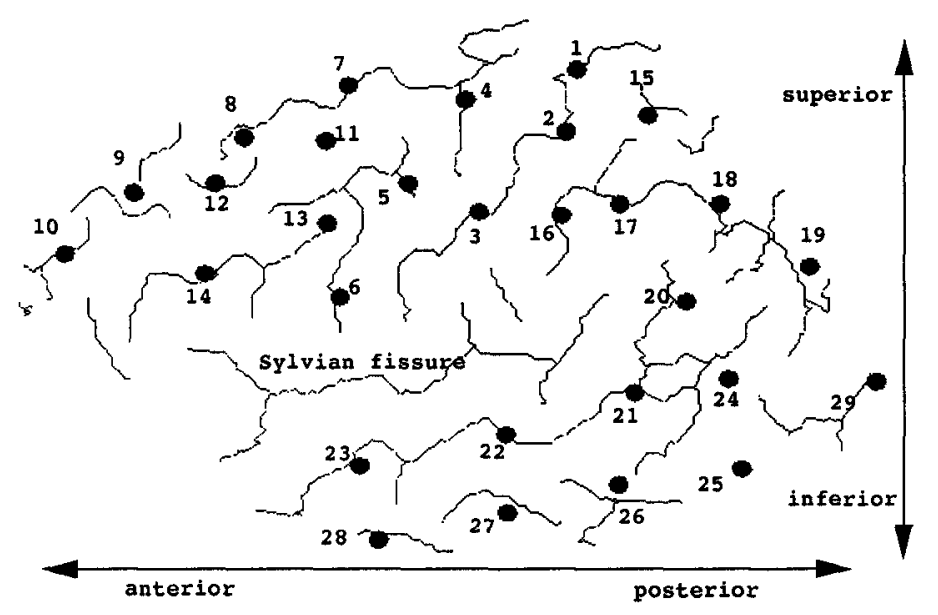

Figure 6: The left hemisphere model

Spatial relations between sulcal basins are defined with respect to the standard coordinate system to which all data sets have been aligned by the preprocessing routine. The relational model describes spatial relationships of three different kinds.

The first type of relation is the "anterior-posterior" relation which describes a basin's position with respect to the coordinate axis along the front/back direction as indicated in figure 6 . For instance, basin 4 is anterior with respect to basin 2 , but posterior to basin 7 . This relation can be easily computed from the basin centroid's address as it is directly related to one of the three coordinate axes of the standard coordinate system.

Likewise, we define a relation called "superior/inferior" (top/bottom) which is determined by a direction orthogonal to the first. The third direction aligned along the third principal axis of the coordinate system is less interesting as the brain extends mostly along the first two axes so that it has less discriminative power.

Instead, we use another relation called "sulcal connectedness" which describes whether the ridge that separates two adjacent basins is "strong" or not. More precisely, two basins are said to be "connected" if the ridge between them is lower than a given threshold. Note that the height of the separating ridge can be easily determined from the distance transform. However, we use this particular relation quite cautiously, as the sulcal connectedness is quite variable from person to person. Only very few sulcal connections can be definitely established. For instance, the basins belonging to the central sulcus must always be connected.

The three relations defined above are represented by three adjacency matrices where each entry in a matrix describes the degree to which the relation between any two basins holds. At present, we distinguish between three degrees: "0" (the relation does not hold), "1" (unknown) and "2" (the relation holds). For instance, basin 5 is always superior to basin 6 . However, it is unknown whether it is superior or inferior to basin 3 , because in some brains it is superior to 
basin 3 whereas in others it is inferior. The adjacency matrix representing the connectedness relation contains mostly "unknowns". However, it is useful in establishing the central sulcus.

\section{Graph matching}

Sulcal basins of some data set can now be labelled by matching them against the model described in the previous section. The technique we use for this purpose is an adaptation of the widely used association graph matching [20, pp.365 ff] which will be explained in the following.

The sulcal basin model described in the previous section yields a graph

$$
G_{1}=\left(V_{1}, R\right)
$$

where $V_{1}$ denotes the set of 29 nodes which represent the centroids of the model basins. Each node $v \in V_{1}$ has a both a neuroanatomical label and a set of descriptors which include its 3D coordinate in the standard coordinate system and its average size or depth. The relational model $R$ is represented by the list of adjacency matrices as defined above.

Likewise, each new data set is represented by a graph

$$
G_{2}=\left(V_{2}, R\right)
$$

where $V_{2}$ is a list of nodes which represent the centroids of the sulcal basins which have been extracted from the data set using the algorithm described in section 2. The spatial relationships between these basins are documented in the list of adjacency matrices contained in $R$.

The association graph

$$
A=(U, S)
$$

contains possible associations between the two graphs $G_{1}$ and $G_{2}$ such that the neuroanatomical labellings of $G_{1}$ are inherited by $G_{2}$ while the constraints imposed by the adjacency matrices are preserved. Nodes in the association graph represent possible matches. More precisely, a node $u$ of the association graph is a pair $u=\left(v_{1}, v_{2}\right) \in U$ where $v_{1} \in V_{1}$ and $v_{2} \in V_{2}$ such that the distance

$$
d=\left\|v_{1}-v_{2}\right\|
$$

does not exceed a given threshold. In our experiments, we used a threshold value of $15 \mathrm{~mm}$. As a further restriction, we only allowed three possible matches for each model node so that in cases where more than three nodes of $G_{2}$ were within a $15 \mathrm{~mm}$ radius of a model node we only admitted the three closest nodes. Such restrictions help to reduce the complexity.

Two nodes in the association graph are linked by an edge if they exhibit compatible spatial relationships. More precisely, two nodes $a=\left(v_{1}, v_{2}\right)$ and $b=\left(u_{1}, u_{2}\right)$ are connected if either

$$
R\left(v_{1}, u_{1}\right)=R\left(v_{2}, u_{2}\right) \neq 1, \text { or } R\left(v_{1}, u_{1}\right)=1 .
$$


Thus, links in the association graph enforce spatial constraints as imposed by the adjacency matrices. If the spatial relationship between any two basins is known (i.e. $R\left(v_{1}, u_{1}\right) \neq 1$ ), then compatibility only holds iff the spatial relationship is the same in both the model graph and the data graph. If the spatial relationship is not known (i.e. $R\left(v_{1}, u_{1}\right)=1$ ) then the link is established anyhow so as not to impose unnecessary constraints.

The process of finding matches between the model graph and the data graph consists in finding maximal cliques in the association graph. A clique is defined as a subset $C$ of nodes such that any two nodes of $C$ are linked by an edge. A maximal clique is a clique which cannot be extended without destroying its clique property.

In the context of graph matching, a clique in the association graph represents a set of matches between model and data graph such every pair of nodes obeys the spatial constraints. If such a clique is maximal, it contains the largest possible number of matches.

Note that there may be more than one maximal clique in the association graph, so that we need to define a criterion that selects a "best" maximal clique. In our experiments, we used the following function to evaluate the quality of a clique:

$$
f(M)=\sum_{(u, v) \in M} \sum_{\left(u^{\prime}, v^{\prime}\right) \in M}\left\|\left(\left\|u-u^{\prime}\right\|-\left\|v-v^{\prime}\right\|\right)\right\|
$$

where $M \subset U$ denotes a clique in the association graph, and $u, u^{\prime}, v, v^{\prime}$ denote spatial addresses of nodes in the graphs $G_{1}$ and $G_{2}$, respectively. The function $f(M)$ measures how well distances between any two nodes in the model graph agree with distances in the data graph. It attains a low value if distances are well preserved, and a high value otherwise.

A large number of algorithms for finding maximal cliques are known from the literature [21]. In our experiments, we used the algorithm described in [20, p. 367]. Regardless of which particular algorithm we use, the problem of finding maximal cliques in a graph is in principal of exponential complexity. Therefore, it is not feasible to seek cliques in an association graph using all 29 basin labels at the same time. We circumvent this problem by following an incremental approach. We begin by first identifying the most prominent and least variable sulci which are the central, precentral, lower postcentral and the anterior intraparietal sulci. We then proceed by identifying the remaining sulci belonging to the frontal lobe, the parietal lobe and finally the temporal lobe, so that the entire identification process is split into four stages. At each stage, approximately eight basin labels are processed.

In spite of the fact that we do not process all labels at the same time, we can still enforce the entire set of constraints across all labels so that the resulting clique obeys all constraints. This is possible as the clique finding method we use supports incremental updates. 


\section{Experiments}

Our input data consisted of T1-weighted magnetic resonance images (MRI) of healthy volunteers. The spatial resolution between planes was approx. $1.5 \mathrm{~mm}$ and the within-plane resolution was set to approx. $0.95 \mathrm{~mm} \times 0.95 \mathrm{~mm}$. The images were subsequently resampled to obtain isotropic voxels of size $1 \mathrm{~mm} \times 1 \mathrm{~mm} \times$ $1 \mathrm{~mm}$ so that each data set contained 160 slices with $200 \times 160$ pixels in each slice. As noted before, all data sets were rotated into a standard coordinate system. In addition, we applied an automatic procedure to extract brain from non-brain material. For a more detailed description of our preprocessing procedure see [22].

Our sulcus detection method was initially applied to four MR data sets so that the unary model could be generated. This model was then used to label sulcal basins which had been extracted from 17 other data sets. None of these 17 data sets had been used for the unary model. So far, we have only processed left hemispheres.

The graph matching algorithm produced maximal cliques which on the average contained 25.4 out of 29 possible matches. In two cases, only 21 and 22 matches were found, in all other cases at least 25 matches were found. An average of 1.1 of the matches found were false. Figure 7 shows a few results.
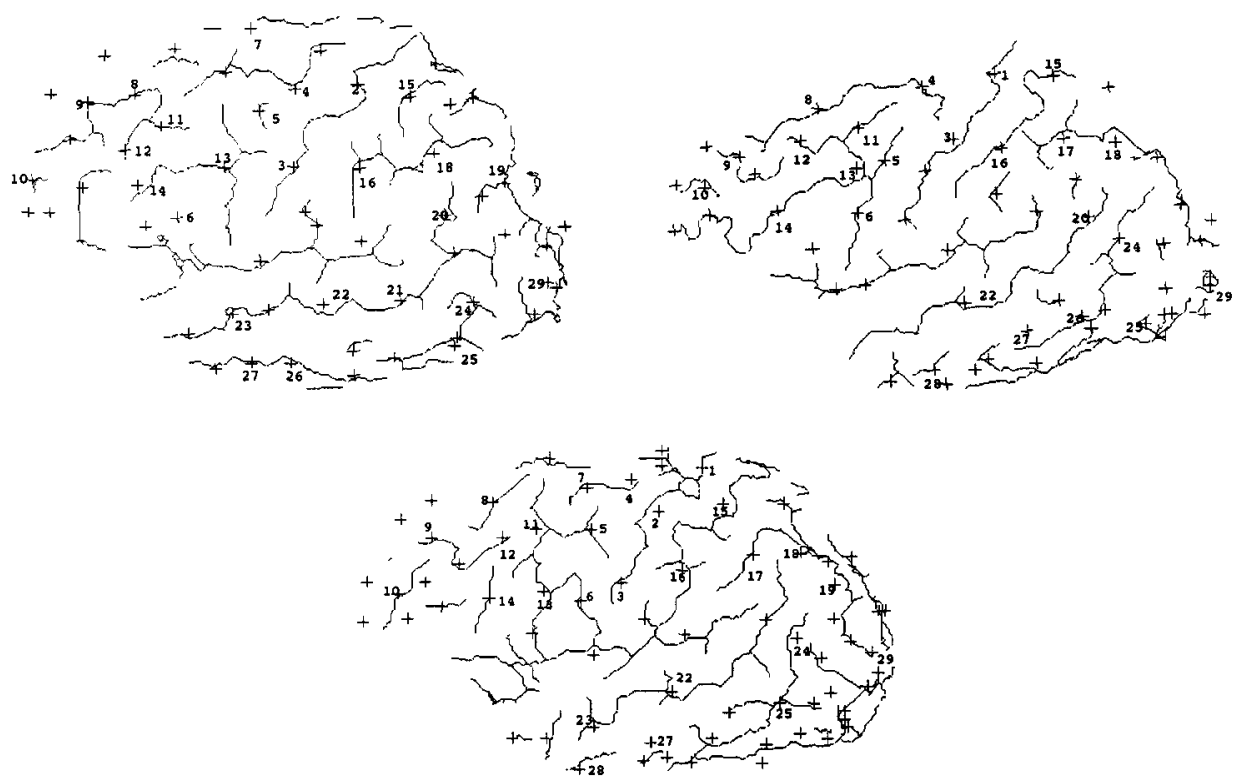

Figure 7: Results of the automatic labelling process

The computation time for the basin identification procedure was approximately 100 seconds. The automatic labelling procedure took approximately 37 seconds. 


\section{Discussion}

We have presented a method that automatically detects and attributes neuroanatomical names to substructures of the cortical folds which we call sulcal basins using magnetic resonance data of healthy human brains. We believe that our method is relevant in the context of human brain mapping because it helps to identify brain loci in a way that is anatomically meaningful and easily reproducible across many subjects so that inter-personal comparisons become possible.

Future work will focus on the following aspects. Firstly, we intend to extend the list of anatomical labels so that brain loci which are not modelled at present (the Sylvian fissures, the medial and basal sulci and some tertiary sulci) can be identified as well. The aim will be to obtain a complete parcellation of the human cortex. At present, the method covers most of the lateral brain surface.

The number of basins varies somewhat between subjects. Therefore, there is some ambiguity in the identification process. For instance, the superior frontal sulcus (basins $7,8,9,10$ ) sometimes contains one basin more or less than our model. In future work, we intend to modify our method so that deviations from the standard model can be dealt with. However, in our experiments so far we found that deviations from the standard model are surprisingly small.

Another aspect of our future work will of course be to improve the accuracy and reliability of the method. At present, the method identifies roughly 85 percent of the sulcal basins with one or two erroneous matches. Improvements can be achieved in several ways. Firstly, the unary model which contains average voxel addresses of the model basins should be incrementally updated as new data sets are processed so that the model begins to "learn". Secondly, other types of spatial relationships between basins will be added. And thirdly, the graph matching procedure will be extended to include probabilistic reasoning.

\section{References}

1. M. Ono, S. Kubik, C.D. Abernathy. Atlas of the cerebral sulci. Georg Thieme Verlag, Stuttgart, New York, 1990.

2. J.C. Maziotta, A.W. Toga, A. Evans, P. Fox, J. Lancaster. A probabilistic atlas of the human brain: theory and rationale for its development. Neuroimage, 2:89-101, 1995.

3. P. Thompson, A.W. Toga. A surface-based technique for warping threedimensional images of the brain. IEEE Transactions on Medical Imaging, 15(4):402-417, 1996.

4. P.M. Thompson, D. MacDonald, M.S. Mega, C.J. Holmes, A.C. Evans, A.W. Toga. Detection and mapping of abnormal brain structure with a probabilistic atlas of cortical surfaces. Journal of Computer Assisted Tomography, 21(4):567-581, 1997.

5. G. Rizzo, P. Scifo, M.C. Gilardi, V. Bettinardi, F. Grassi, S. Cerutti, F. Fazio. Matching a computerized brain atlas to multimodal medical images. NeuroImage, 6(1):59-69, 1997.

6. S. Sandor, R. Leahy. Surface-based labeling of cortical anatomy using a deformable atlas. IEEE Transactions on Medical Imaging, 16(1):41-54, Feb. 1997. 
7. A. Guéziec, N. Ayache. Smoothing and matching of 3-d space curves. International Journal of Computer Vision, 12(1):79-104, 1994.

8. J. Declerck, G. Subsol, J.-P. Thirion, N. Ayache. Automatic retrieval of anatomical structures in 3D medical images. In N. Ayache, editor, Computer Vision, Virtual Reality and Robotics in Medicine, pages 153-162, Nice, France, April 1995. Springer Lecture Notes, 905.

9. J.-F. Mangin, V. Frouin, I. Bloch, J. Régis, and J. López-Krahe. From 3-D magnetic resonance images to structured representations of the cortex topography using topology preserving deformations. Journal of Mathematical Imaging and Vision, $5(4): 297-318,1995$.

10. J.F. Mangin, V. Frouin, J. Regis, I. Bloch, P. Belin, Y. Samson. Towards better management of cortical anatomy in multi-modal multi-individual brain studies. Physica medica, XII(Supplement 1):103-107, June 1996.

11. A. Manceaux-Demiau, J.F. Mangin, J. Regis, Olivier Pizzato, V. Frouin. Differential features of cortical folds. In CVRMed, Grenoble, France, 1997.

12. M. Valliant, C. Davatzikos, R.N. Bryan. Finding 3D parametric representations of the deep cortical folds. In Proc. Mathematical Methods in biomedical image analysis (MMBIA 96), pages 151-157, San Francisco, CA, June 1996. IEEE Computer Society.

13. M. Valliant, C. Davatzikos. Mapping the cerebral sulci: application to morphological analysis of the cortex and to non-rigid registration. In Int. Conf. on Information Processing in Medical Imaging (IPMI 97), pages 141-154, Poultney, Vermont, USA, June 9-13 1997.

14. M. Näf, O. Kübler, R. Kikinis, M.E. Shenton, G. Szekely. Characterization and recognition of $3 \mathrm{D}$ organ shape in medical image analysis using skeletonization. In Proc. Mathematical Methods in biomedical image analysis (MMBIA 96), pages 139-150, San Francisco, CA, June 1996. IEEE Computer Society.

15. G. Lohmann. A new approach to white matter segmentation in MR images. Technical report, Max-Planck-Institute of Cognitive Neuroscience, Leipzig, Germany, 1997.

16. P. Maragos, R.W. Schafer. Morphological systems for multidimensional signal processing. Proc. of the IEEE, 78(4):690-709, 1990.

17. G. Borgefors. Distance transforms in arbitrary dimensions. Computer Vision, Graphics, and Image Processing, 27:321-345, 1984.

18. P.T. Fox, J.S. Perlmutter, M.E. Raichle. A stereotactic method of anatomical localization for positron emission tomography. Journal of Computer Assisted Tomography, 9(1):141-153, Jan./Feb. 1985.

19. G.Lohmann. Extracting line representations of sulcal and gyral patterns in MR images of the human brain. Technical report, Max-Planck-Institute of Cognitive Neuroscience, Leipzig, Germany, July 1997.

20. D. Ballard, C.M. Brown. Computer Vision. Prentice Hall, Englewood Cliffs, NJ, 1982.

21. E.A. Akkoyunlu. The enumeration of maximal cliques of large graphs. SIAM J. Comput., 2(1), March 1973.

22. F. Kruggel, G.Lohmann. Automatical adaptation of the stereotactical coordinate system in brain MRI data sets. In J. Duncan, editor, Int. Conf. on Information Processing in Medical Imaging (IPMI 97), Poultney, Vermont, USA, June 9-13 1997. 